\title{
Electrocardiogram measurements in water using poly(3,4-ethylene dioxythiophene):poly(styrene sulfonate) nanosheets waterproofed by polyurethane film
}

\author{
Sho Mihara, Graduate School of Advanced Science and Engineering, Waseda University, Tokyo 162-8480, Japan; Waseda Research Institute for Science \\ and Engineering, Waseda University, Tokyo 169-8555, Japan \\ Hui-Lin Lee, School of Chemical \& Life Sciences, Singapore Polytechnic, 500 Dover Road, Singapore 139651, Singapore \\ Shinji Takeoka, Waseda Research Institute for Science and Engineering, Waseda University, Tokyo 169-8555, Japan; Faculty of Science and Engineering, \\ Waseda University, Tokyo 169-8555, Japan
}

Address all correspondence to Shinji Takeoka at takeoka@waseda.jp

(Received 3 June 2020; accepted 11 September 2020)

\begin{abstract}
Waterproof bioelectrodes enable long-term biological monitoring and the assessment of performances of athletes in water. Existing gel electrodes change their electrical properties even when covered with a waterproof film. Here, the authors present the poly(3,4-ethylene dioxythiophene):poly(styrene sulfonate) (PEDOT:PSS)/poly(styrene-butadiene-styrene) (SBS) bi-layer nanosheet and waterproof film for a comfortable waterproof bioelectrode. PEDOT:PSS/SBS is fully foldable with a conductivity loss of only $5 \%$. This foldable nanosheet electrode provides a reliable electrical connection between the skin and the wire. The waterproof film-covered bioelectrode enables continuous monitoring of electrocardiograms in water, showing a signal-to-noise ratio of $21.5 \mathrm{~dB}$ for the $\mathrm{R}$ wave and $17.5 \mathrm{~dB}$ for the $\mathrm{T}$ wave, comparable to atmospheric measurements, and sensing a change in heart rate from 79 to 131 bpm during bathing.
\end{abstract}

\section{Introduction}

A recent development in electronic manufacturing technology has significantly contributed to the miniaturization and mass production of medical devices. ${ }^{[1]}$ Research on the daily sensing of biological signals and markers using advanced medical devices is currently receiving great attention ${ }^{[2-4]}$ to promote "healthy life expectancy" with the extension of life expectancy worldwide. $^{[5]}$ With infectious diseases such as COVID-19, which is currently spreading worldwide and the ensuing "emergency lockdowns implemented by various Governments," individuals prone to chronic diseases may face fatal outcomes in their home settings if disease signs and symptoms are unchecked. The detection of sudden changes in physical conditions can be achieved online with biosensing devices, with appropriate follow-up treatments suggested. ${ }^{[6]}$

Therefore, a method for reliable electrocardiogram (ECG) measurements is desired for the early detection of cardiovascular diseases, which seriously threaten our lives. ${ }^{[7]}$ The primary method of measuring ECG is to apply a conductive hydrogel electrode to the skin surface, but it is not easy to use such an electrode during daily life because gel swells with water. The swollen gel changes its electrical properties and easily detaches from the skin, preventing the continuation of ECG measurements.

This problem may be mitigated with the development of a waterproof ECG measurement. Continuous ECG measurement during bathing, which has the highest risk of myocardial infarction in daily life, is an effective means to prevent sudden death in the elderly. Also, waterproof ECG measurement can be utilized for the performance evaluation of athletes during swimming and biopotential survey of marine organisms, expanding the area of biological sensing.

Reyes et al. reported that water permeation into the gel electrode reduced the ECG signal quality and proposed a new hydrophobic electrode. ${ }^{[8]}$ However, the thick electrode with a thickness of $2 \mathrm{~mm}$ must be fixed to the skin with a surgical tape, which deteriorates the Quality of Life of the subjects.

In this study, we report a comfortable and waterproof ECG measurement system using conductive nanosheets composed of poly(3,4-ethylene dioxythiophene):poly(styrene sulfonate) (PEDOT:PSS) and poly(styrene-butadiene-styrene) (SBS) as an electrode. The assembly is protected by covering it with a waterproof polyurethane film. Electrodes fabricated with PEDOT:PSS have excellent electrical properties to record high-quality electrophysiological signals ${ }^{[9,10]}$ when applied to skin contact electrodes and implantable devices. However, PEDOT:PSS does not have tackiness, unlike existing conductive hydrogels. Therefore, in order to adhere PEDOT:PSS to the skin, improved adhesion is required. Wang et al. reported biosignal acquisition using a conductive hydrogel as a body surface electrode. ${ }^{[11]}$ Tsukada et al. developed a thread-like electrode made of PEDOT:PSS and silk fibers and reported biosignal acquisition. ${ }^{[12]}$ 
We have reported a technique for making an ultra-thin PEDOT:PSS film (PEDOT:PSS nanosheet) with significantly reduced bending stiffness and firm bonding to skin. ${ }^{[1-15]}$ However, handling the PEDOT:PSS nanosheet requires the support of an insulating elastomer nanosheet with high strength. One side of the bi-layered nanosheet (PEDOT: PSS nanosheet/elastomer nanosheet) has conductivity. Although the biological signal could be detected on the skin with this nanosheet electrode, the connection of wiring for signal analysis was a problem. In order to make the conductive surface of the nanosheet electrode contacted with the wiring, the wiring had to be sandwiched between the nanosheet electrode and the skin. A huge gap in size and stiffness between the nanosheet electrode and the wiring resulted in a frangible structure disturbed by even a slight wiring movement.

In this study, we folded the bi-layered nanosheet, which has a conductive side and an insulating side to make the insulating side inside. The folded nanosheet had conductive surfaces and enabled a good connection with a connector. The folded nanosheet electrode's (FNE) thickness was less than $1 \mu \mathrm{m}$, that was comfortable on the skin, and could be enclosed fully by a polyurethane waterproof film. Here, we evaluated the waterproofing's effectiveness over FNE on the skin covered with the polyurethane film for ECG monitoring simulated bathing conditions. If successful, this folded nanosheet assembly could be applied to individuals suffering from chronic heart disease, thus reducing fatalities.

\section{Materials and methods Materials}

Poly(ethylene terephthalate) (PET) film substrate, Lumirror 25T60, was purchased from Panac Co. (Tokyo, Japan). PEDOT:PSS aqueous dispersion, Clevios PH 1000 (1:2.5 PEDOT:PSS ratio), was purchased from H.C. Starck GmbH (Leverkusen, Germany). Poly (vinyl alcohol) (PVA; Mw $=\sim 22,000$ ), ethyl acetate, and tetrahydrofuran (THF) were purchased from Kanto Chemical Co. (Tokyo, Japan). SBS $(\mathrm{Mw}=\sim 140 \mathrm{kDa})$, polystyrene (PS; Mw 280000), and Zonyl® FS-300 fluorosurfactant (Zonyl) were purchased from Sigma-Aldrich, Co. (St. Louis, MO, USA). Dipropylene glycol (DPG) was purchased from Tokyo Chemical Industry Co. (Tokyo, Japan). A nylon mesh sheet, CLV-PTM-33 (Opening $40 \mu \mathrm{m}$, Thread diameter 40 $\mu \mathrm{m}$, Open area 25\%), was purchased from Nippon-clever Co. (Aichi, Japan). A copper sputtered conductive adhesive film (Conductive-Tape), 7836XCSB, was purchased from Sekisui Chemical Co. Ltd (Tokyo, Japan). A polyurethanebased transparent adhesive plaster, Cathereeplus ${ }^{\mathrm{TM}}$, was purchased from Nichiban Co., Ltd (Tokyo, Japan). An artificial skin model, Bioskin plate, was purchased from Beaulax Co., Ltd (Saitama, Japan). A disposable gel electrode, Vitrode F, was purchased from NIHON KOHDEN Co. (Tokyo, Japan). (a)

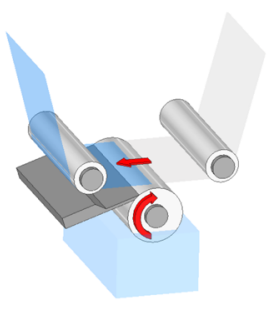

(b)
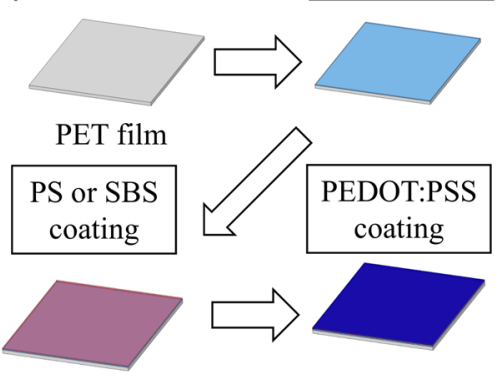

(c) Adhesive tape flame

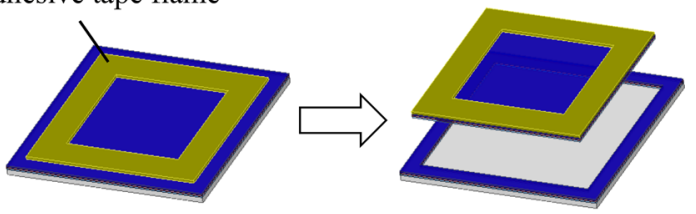

(d)

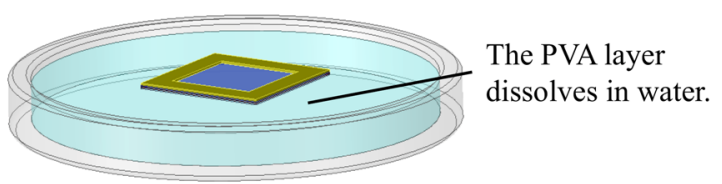

(e)
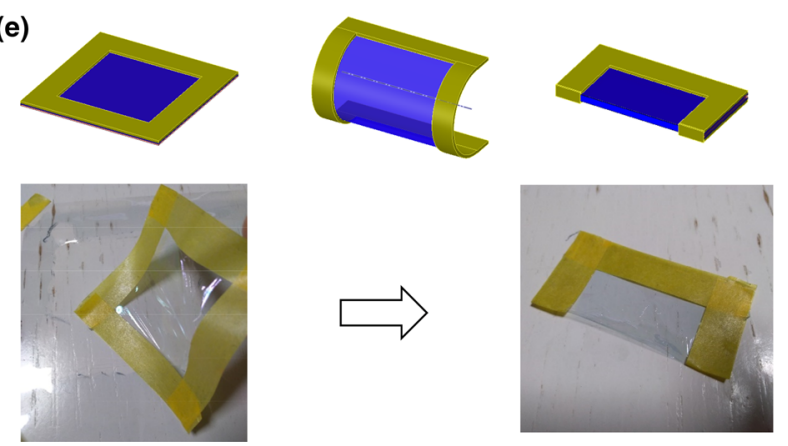

Figure 1. Schematic illustration of (a) the micro gravure coating technique, (b) the scheme of processing for the conductive nanosheet, (c) the physical separation of the conductive nanosheet from the PET substrate, (d) the dissolution of the water-soluble sacrificial layer, and (e) schematic illustration and picture of the folding of the conductive nanosheet.

\section{Fabrication of PEDOT:PSS/SBS, PEDOT:PSS/PS nanosheets}

PEDOT:PSS/SBS and PEDOT:PSS/PS conductive nanosheets were fabricated by a roll-to-roll gravure printing method as shown in Fig. 1(a). First, a water-soluble sacrificial layer of PVA was formed on a PET substrate by gravure-coating a PVA aqueous solution $(50 \mathrm{mg} / \mathrm{mL})$. On the dried PVA sacrificial layer, an SBS solution in THF $(20 \mathrm{mg} / \mathrm{mL})$ or a PS solution in THF $(20 \mathrm{mg} / \mathrm{mL})$ was gravure-coated to form an SBS nanosheet or a PS nanosheet with a thickness of $116 \pm 13$ and $118 \pm$ $11 \mathrm{~nm}$, respectively. Next, a PEDOT:PSS aqueous dispersion (Clevios PH 1000) was mixed with DPG (5 v/v\%) and Zonyl $(1 \mathrm{v} / \mathrm{v} \%)$, stirred for $8 \mathrm{~h}$ at room temperature, and then gravurecoated over the SBS layer or the PS layer to form a PEDOT: 
PSS nanosheet as shown in Fig. 1(b) with a thickness of $189 \pm$ 20 and $188 \pm 31 \mathrm{~nm}$, respectively. A thermal treatment $\left(140^{\circ} \mathrm{C}\right.$, $15 \mathrm{~min}$ ) was carried out on the rolled sample (width: $12 \mathrm{~cm}$; length: 5 $\mathrm{m}$ ) to change the state of PEDOT:PSS from colloidal particles to a solid film ${ }^{[16]}$ and enhance conductivity. The conductivity of PEDOT:PSS/SBS/PVA/PET or PEDOT:PSS/PS/ PVA/PET was measured by the four-terminal method resistance measuring apparatus (Loresta-GP, Mitsubishi chemical analytech Co., Ltd, Kanagawa, Japan).

\section{Stability of the PEDOT:PSS/SBS nanosheets against folding}

By attaching an adhesive tape frame on the top of the PEDOT: PSS layer, the $40 \times 40 \mathrm{~mm}$ PEDOT:PSS/SBS/PVA or PEDOT: PSS/PS/PVA tri-layered film was physically peeled off from the PET substrate as shown in Fig. 1(c). With the help of mechanical support with the tape frame, a free-standing PEDOT:PSS/SBS or PEDOT:PSS/PS bi-layered conductive nanosheet was obtained by dissolving the PVA layer in water as shown in Fig. 1(d). The conductivity of PEDOT:PSS/SBS or PEDOT:PSS/PS was measured by contacting digital multitester probes (B35T, Fujian Lilliput Optoelectronics Technology Co., Ltd, Zhangzhou, China) with the edge of their sheets. The nanosheet was then folded into two layers by attaching the SBS layer or the PS layer to expose PEDOT: PSS on both the top and bottom layers as shown in Fig. 1(e). The folded bi-layered nanosheet with a $40 \times 20 \mathrm{~mm}$ size was collected on a nylon mesh as a temporary substrate, and the tape frame was cut and removed. The conductivities of PEDOT:PSS/SBS and PEDOT:PSS/PS folded nanosheets were measured by contacting a digital multi-tester probe with each front and back edge as shown in Fig. 2(a). Moreover, the frequency characteristics of PEDOT:PSS/SBS folded nanosheet were measured by using the chemical impedance meter (HIOKI 3532-80; HIOKI E.E. Co., Nagano, Japan). The two PEDOT:PSS/SBS folded nanosheets were placed in contact with each other on their conductive surfaces, and the impedance between them was measured in the frequency range from $10 \mathrm{~Hz}$ to $100 \mathrm{kHz}$.

\section{Waterproof test in vitro}

The waterproof of the PEDOT:PSS/SBS FNE (SBS-FNE) and disposable gel electrode was evaluated in vitro. An artificial skin model was used as a model of skin. First, two Conductive-Tape $(40 \times 10 \mathrm{~mm})$ were attached in parallel to the artificial skin model so that the conductive surface was on top, and the interval between the tapes was $20 \mathrm{~mm}$ as shown in Fig. 3(a). The SBS-FNE was transferred from the mesh sheet to the artificial skin using a small amount of water such that both edges of SBS-FNE overlapped the edges of Conductive-Tape. Two metal wirings were then attached to each Conductive-Tape, where the SBS-FNE was not attached. After drying at room temperature for $30 \mathrm{~min}$, the whole system of SBS-FNE, Conductive-Tape, and metal wirings was fixed with a $50 \times 60 \mathrm{~mm}$ Cathereeplus ${ }^{\mathrm{TM}}$. The digital multi-tester (a)
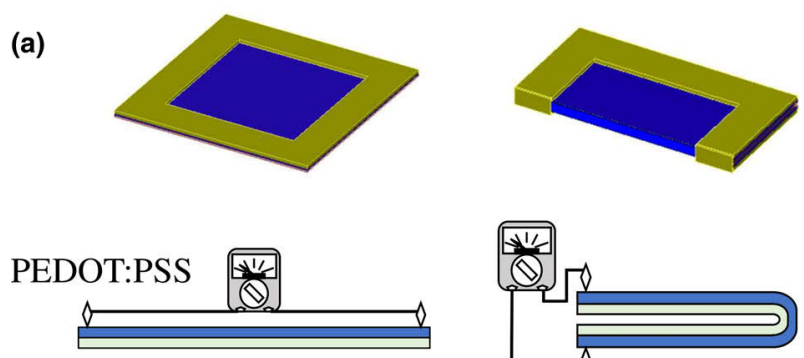

SBS or PS

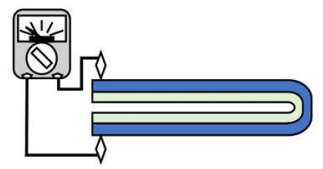

Figure 2. (a) Schematic illustration of measurement points for evaluate conductivity when before and after folding conductive nanosheet and (b) conductivity change of PEDOT:PSS/SBS and PEDOT:PSS/PS by folding.

and metal wirings were connected, and the resistance values were recorded. The system on the artificial skin was immersed in the warm water of $40-43{ }^{\circ} \mathrm{C}$ for $1 \mathrm{~h}$ with reference to a previous study. ${ }^{[17]}$ The change in resistance values of SBS-FNE was recorded. The system was pulled up with the artificial skin from the warm water, and the moisture was wiped off. The resistance value of SBS-FNE was measured after $30 \mathrm{~min}$ of drying at room temperature. The resistance change of the disposable gel electrode during immersion in warm water was also recorded in the same protocol. The warm water immersion tests were conducted for SBS-FNE and the disposable gel electrode without the Cathereeplus ${ }^{\mathrm{TM}}$ urethane dressing films to confirm waterproofing ability.

\section{Recording of ECG signals and evaluation}

All measurements of ECG signal monitoring were carried for one (S.M.) of authors. The SBS-FNEs supported on nylon mesh substrates were transferred to the following sites under the right clavicle, under the left clavicle, and at the lower left abdomen, as shown in Fig. 4(a), with the aid of a small amount of water. The insulating metal wiring was connected to the edge of each SBS-FNE via Conductive-Tape as shown in Supplementary Fig. S5. The SBS-FNE, Conductive-Tape, 
(a)
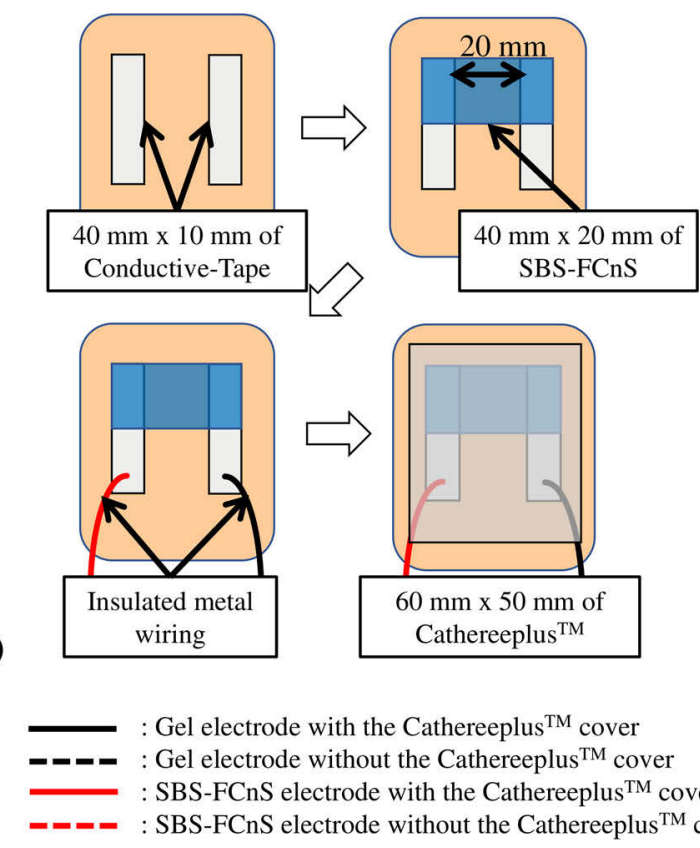

: Gel electrode with the Cathereeplus ${ }^{\mathrm{TM}}$ cover : Gel electrode without the Cathereeplus ${ }^{\mathrm{TM}}$ cover : SBS-FCnS electrode with the Cathereeplus ${ }^{\mathrm{TM}}$ cover : SBS-FCnS electrode without the Cathereeplus ${ }^{\mathrm{TM}}$ cover

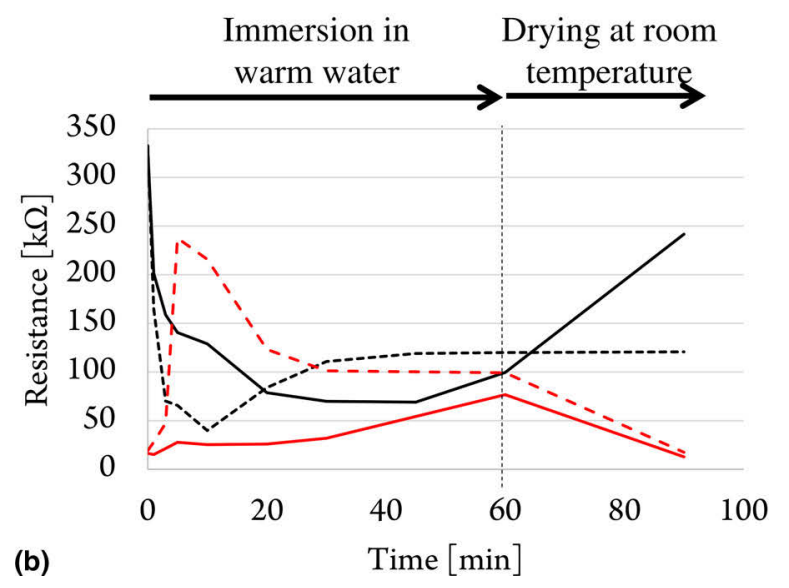

Figure 3. (a) Schematic illustration of the sample preparation procedure for the waterproof test. (b) The resistance change of electrodes in warm water. Black solid line shows the gel electrode coated by Cathereeplus ${ }^{\mathrm{TM}}$. Black dot line shows the gel electrode without cover. Red solid line shows SBS-FNE coated by Cathereeplus ${ }^{\mathrm{TM}}$. Red dot line shows SBS-FNE without cover.

and insulating metal wirings were covered with Cathereeplus ${ }^{\mathrm{TM}}$, and the whole system was fixed on the skin. The other end of the insulating metal wirings was related to an amplifier circuit in Bitalino (Plux-Wireless Biosignals S. A., Portugal). The amplifier circuit was affixed to the main circuit of Bitalino, which was composed of a control circuit, battery, and Bluetooth device. The ECG signal was acquired at $100 \mathrm{~Hz}$ and amplified about 316,000 times. ${ }^{[18]}$ The amplified ECG signal data were sent to PC via Bluetooth using open-source software (Opensignals, Plux-Wireless Biosignals S. A., Portugal).

The ECG data were analyzed using Excel for Office365 (Microsoft Co., WA, USA) and Matlab 2019b (The Math Works, MA, USA). The heart rate and signal-to-noise ratios
(SNRs) were calculated from each measurement data. The heart rate was calculated as the average of R-R intervals. The SNRs were calculated and compared simply by the following equation:

$$
\mathrm{SNR}[\mathrm{dB}]=10 \log _{10} \frac{S}{N}
$$

where $S$ is the power of the signal, and $N$ is the power of the noise. In this study, $S$ was simply calculated from the maximum of each wavelength of the ECG signal. $N$ was calculated from the baseline of the ECG signal.

\section{ECG signals monitoring during bath}

ECG signal monitoring was conducted during bath and compared before and after a bath. At first, ECG signal recording was conducted for 10 min before taking a bath with keeping the other conditions (room temperature: $24.5^{\circ} \mathrm{C}$, humidity: $46 \%$ ). The body surface temperature was visualized with a thermographic camera (CPA-E60A, FLIR systems Inc., OR, USA). The bath with a water temperature of $40-43{ }^{\circ} \mathrm{C}$ was prepared. The ECG signal was recorded for $10 \mathrm{~min}$ during the bath. Immediately after leaving the bath, the body was wiped, and body surface temperature was then measured. ECG measurements were recorded up to $10 \mathrm{~min}$ after bathing.

\section{Results and discussion Stability of the bi-layered PEDOT:PSS/SBS nanosheet against folding}

A three-layer thin film of PEDOT:PSS/SBS/PVA or PEDOT: PSS/PS/PVA was prepared on PET by a micro gravure coater as shown in Figs. 1(a) and 1(b). These conductive films showed a conductivity of 98 and $110 \mathrm{~S} / \mathrm{cm}$, respectively. Generally, PEDOT:PSS is too fragile to be a self-standing thin film. The plastic layer supports the PEDOT:PSS layer, resulting in a selfstanding conductive nanosheet. ${ }^{[13]}$ On the other hand, the insulating surface of those nanosheets did not show detectable conductivity. These thin films were mechanically peeled off and used as nanosheets by dissolving the water-soluble sacrificial layer as shown in Figs. 1(c) and 1(d) and completely folded as shown in Fig. 1(e).

The FNE is performed to prevent damage at the connection edge with the connector. When the bi-layered nanosheet electrode is used as a body surface electrode, the conductive side must contact the skin. However, the conductive side also needs to be connected to metal wiring, linked to the amplifier circuit.

In this study, the conductive nanosheet was connected to metal wiring via Conductive-Tape to reduce the physical property gap while firmly bonding. This Conductive-Tape has a conductive adhesive layer, a copper sputter layer, and a PET film layer as shown in Supplementary Fig. S1, and is suitable as wiring for connecting to PEDOT:PSS that does not have adhesiveness. The contact resistance between ConductiveTape and PEDOT:PSS was only $1.2 \times 10^{-3} \Omega / \mathrm{m}^{2}$. However, 
(a)

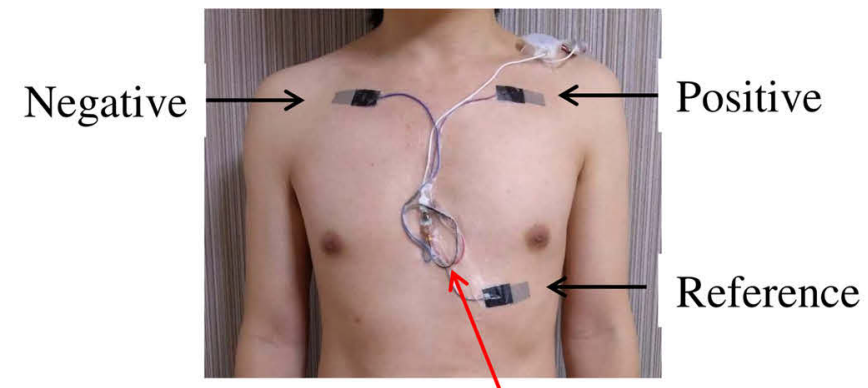

All electrodes, Conductive-Tape, wire and amplifier circuit are fixed to the skin with Cathereeplus ${ }^{\mathrm{TM}}$.

(b)

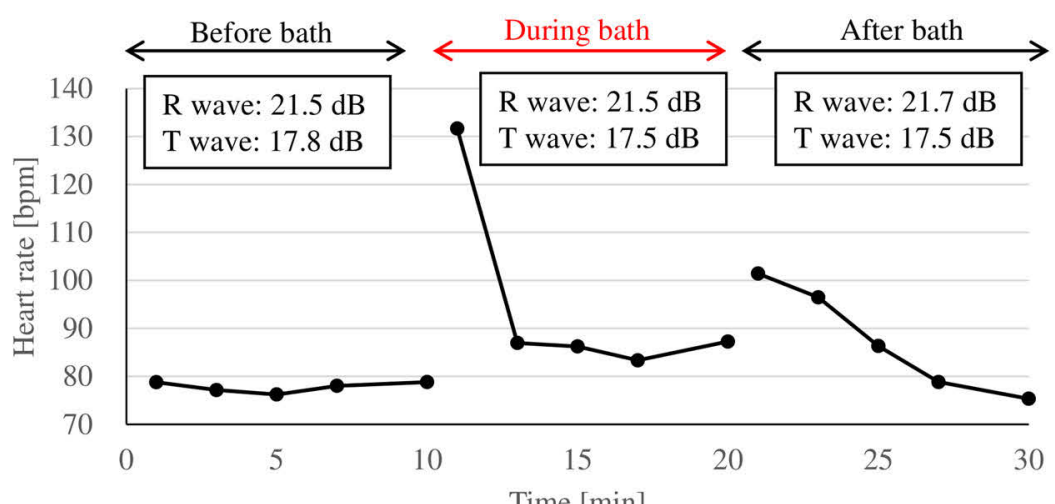

After bath

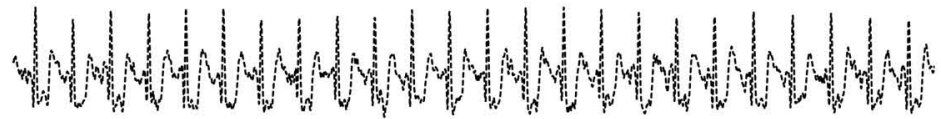

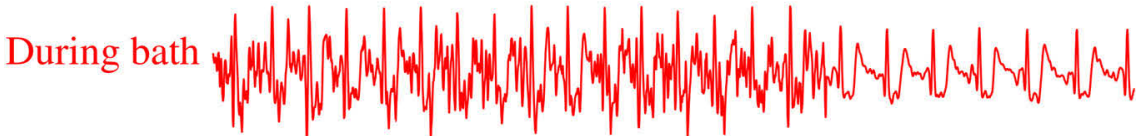

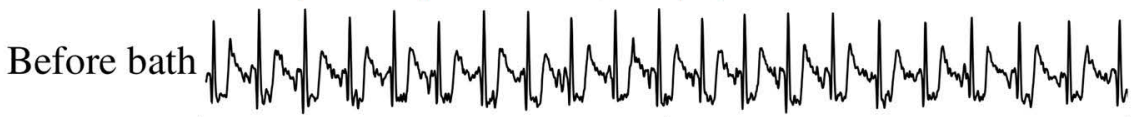

(c)
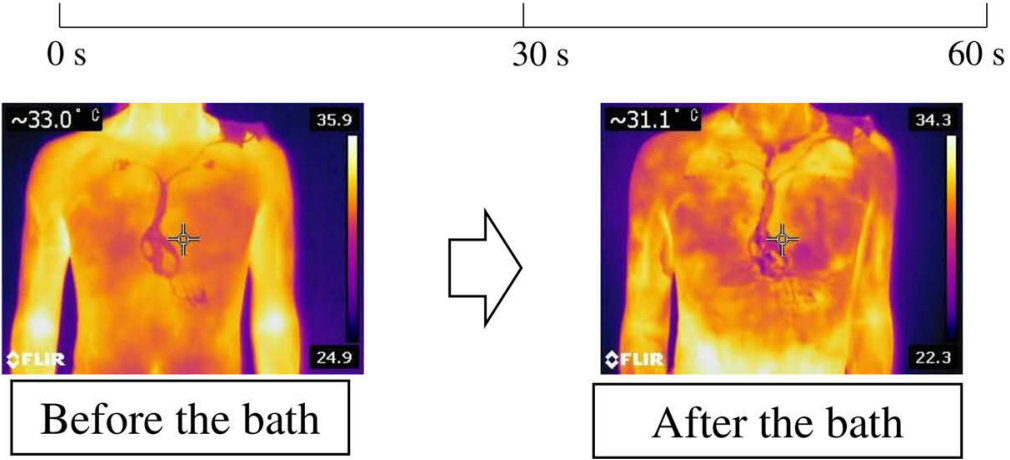

(d)

Figure 4. (a) The picture of the whole system for ECG measurement by using SBS-FNEs. (b) The changes in heart rate before, during, and after bathing, and signal SNR during each phase. (c) Part of ECG before, during, and after bathing. (d) The body surface temperature measurement before and after bathing using a thermography camera. 
there is a big difference in thickness between Conductive-Tape and nanosheet; nanosheet is easily destroyed as shown in Supplementary Fig. S2(a).

To solve the thickness gap problem, we propose FNE to ensure that the insulating layer faces inwards and the conductive sides facing outwards. FNE can be directly affixed to the skin surface and connected to a conductive film from FNE to reduce the physical load generated by the thickness gap as shown in Supplementary Fig. S2(b).

Electronic sheet materials are usually subjected to bending tests to prove their flexibility. In general, the bending test is performed by applying a sheet to a curved substrate at a bending radius of a few millimeters. ${ }^{[19]}$ Though $180^{\circ}$ bending is considered hard to achieve by any electronics materials, our PEDOT: PSS nanosheet supported by SBS achieved $180^{\circ}$ bending with keeping their conductivity. The bi-layered PEDOT:PSS/SBS nanosheet was completely folded, and conductivity was compared before and after folding as shown in Fig. 2(a). The conductivity was maintained even after bending with only $5 \%$ reduction as shown in Fig. 2(b). On the other hand, the same test was performed for the folded PEDOT:PSS/PS nanosheet. The conductivity decreased significantly and deteriorated by $75 \%$.

The elastic modulus of SBS nanosheets and PS nanosheets was reported by Sato et al., ${ }^{[20]}$ and there is a significant difference between $0.045 \mathrm{GPa}$ (thickness: $212 \mathrm{~nm}$ ) and $1.19 \mathrm{GPa}$ (thickness: $217 \mathrm{~nm}$ ), respectively. The bending stiffness calculated by the product of the elastic modulus and the moment of inertia of the area suggests that the bending stiffness of the SBS nanosheet is low. Interestingly, in this experiment, it was found that the use of SBS, which has a lower bending stiffness than PS, resulted in less deterioration of the conductivity of PEDOT:PSS. It was suggested that the substrate's flexibility might contribute to the improvement of the flexibility of the PEDOT:PSS film.

The frequency characteristics of SBS-FNE were shown in Supplementary Fig. S1. According to the standard of disposable ECG electrodes (ANSI/AAMI EC12:2000/(R)2015), the resistance value at $10 \mathrm{~Hz}$ should be $3 \mathrm{k} \Omega$ or less. The impedance of SBS-FNE at $10 \mathrm{~Hz}$ is only $1.9 \mathrm{k} \Omega$, and it is suitable as an ECG electrode in terms of impedance characteristics.

The SBS-FNE can be used as nanomaterial with a thickness of about $600 \mathrm{~nm}$ even after folding, and show tight adhesion to the skin surface without any adhesive glue.

\section{Waterproof test in vitro}

Before evaluating the electrodes, resistance values of the artificial skin and Conductive-Tape in warm water were evaluated. The artificial skin did not show conductivity in warm water. The resistance value of Conductive-Tape did not change significantly from $6 \Omega$ even when immersed in warm water. From these results, it was suggested that the electric characteristics of the base material and the wiring were stable during the immersion test.
The procedure and results were shown in Figs. 2(a) and 2(b). With or without the Cathereeplus ${ }^{\text {TM }}$ cover, the gel electrode resistance was significantly reduced immediately after immersion and then increased. The gel electrode without the Cathereeplus $^{\mathrm{TM}}$ cover had a faster change in resistance, reaching $40 \mathrm{k} \Omega$ after $10 \mathrm{~min}$ of immersion, and the gel electrode with the Cathereeplus ${ }^{\mathrm{TM}}$ cover reaching $69 \mathrm{k} \Omega$ after $30 \mathrm{~min}$ of immersion. After that, the gel electrode's resistance value without the cover increased to $121 \mathrm{k} \Omega$, whereas that of the gel electrode with the cover slowly increased to $100 \mathrm{k} \Omega$. The gel electrode without a cover swelled several times its original volume as shown in Supplementary Fig. S4(a) did not return to its original shape even after drying for $30 \mathrm{~min}$, and the resistance value did not change.

The decrease in the resistance value immediately observed after the immersion of the gel electrode is presumed to be due to the improvement of the ion conductivity due to the warming of the gel. The subsequent increase in resistance seems to be due to gel swelling. The resistance of warm water in this experiment was $118 \mathrm{k} \Omega$, which was similar to the gel electrode resistance after swelling.

The swelling was also observed with the covered gel electrode as shown in Supplementary Fig. S4(b). Due to drying, the resistance value tended to return to the original value but did not completely return to the original value. The high moisture permeability of the Cathereeplus ${ }^{\mathrm{TM}}$ cover is expected to swell the gel electrode. The Cathereeplus ${ }^{\mathrm{TM}}$ is generally characterized to have high water vapor permeability, and it has been reported to have a moisture permeability of $6179 \mathrm{~g} / \mathrm{m}^{2} / 24 \mathrm{~h}^{[21]}$

On the other hand, a completely different feature was observed for the SBS-FNE. The resistance value of the SBS-FNE without a Cathereeplus ${ }^{\mathrm{TM}}$ cover significantly increased from 16 to $240 \mathrm{k} \Omega$ immediately after immersion in warm water. After that, the resistance dropped down to 100 $\mathrm{k} \Omega$ and stabilized. On the other hand, no drastic change was observed in the resistance value of the SBS-FNE covered with Cathereeplus ${ }^{\mathrm{TM}}$, and the resistance value gradually increased to only $77 \mathrm{k} \Omega$. Regardless of the presence or absence of the Cathereeplus ${ }^{\mathrm{TM}}$ cover, the resistance of these SBS-FNE recovered as about $17 \mathrm{k} \Omega$, almost the same values before immersion by drying for $30 \mathrm{~min}$.

The SBS-FNE did not decrease resistance immediately after immersion in warm water, which was observed with gel electrodes. Since the conductivity of PEDOT:PSS does not depend only on ionic conduction, the resistance value is more stable than that of the gel electrode. These results support that SBS-FNE is more suitable for use in high-temperature environments than gel electrodes.

The increase in the resistance of the SBS-FNE in warm water would be due to the contact of water to PEDOT:PSS because PEDOT:PSS with water is also applied to humidity sensors. It has also been reported that the resistance decreases when PEDOT:PSS is saturated with water. ${ }^{[22]}$ However, the resistance change of the SBS-FNE caused by contact with water was suppressed by covering with Cathereeplus ${ }^{\mathrm{TM}}$. The 
reason why the resistance value of the SBS-FNE was recovered by drying after removing water is presumed to be that the amount of swelling would be small. The PEDOT:PSS layer of the SBS-FNE is much thinner than the gel layer of the gel electrode, and swells less with water. No swelling was observed on the SBS-FNE without the actual urethane cover as shown in Supplementary Fig. S4(c). These results suggest that the electrical properties of the SBS-FNE are recoverable and suitable for long-term use in daily life.

Besides, the Cathereeplus ${ }^{\mathrm{TM}}$ which covered the gel electrode is bulky. Gaps between the polyurethane cover and skin promote water ingress. Furthermore, the bulky gel electrode is not comfortable for the subject. From the improvement of Quality of Life, the use of the SBS-FNE is excellent for daily monitoring of vital information, including bathing.

In summary, this in vitro test revealed that SBS-FNEs covered with Cathereeplus ${ }^{\mathrm{TM}}$ had higher heat resistance, water resistance, durability, recoverability, and comfortably than gel electrodes.

\section{ECG measurement during the bath}

SBS-FNE with a thickness of only $600 \mathrm{~nm}$ on nylon mesh were transferred to the skin using a small amount of water. By drying SBS-FNE, it was firmly adhered to the skin without adhesive as shown in Supplementary Figs. S5(a) and S5(b). The metal wiring was connected to the SBS-FNE via Conductive-Tape as shown in Supplementary Fig. S5(c) and S6. All electrodes and wiring were covered with Cathereeplus ${ }^{\mathrm{TM}}$ as shown in Fig. 4(a).

Changes in heart rate before, during, and after bathing and SNRs of $\mathrm{R}$ and $\mathrm{T}$ waves are shown in Fig. 4(b). The ECG for $1 \mathrm{~min}$ picked up from the measurement result for $10 \mathrm{~min}$ in each state is shown in Fig. 4(c).

A resting ECG was recorded before bathing. Characteristic signals of ECG were observed with excellent SNRs of 21.5 and $17.8 \mathrm{~dB}$ for $\mathrm{R}$ and $\mathrm{T}$ waves, respectively. At the same time, stable heart rates were recorded in the range of 70-80 bpm, which are the average heart rates of a healthy adult male. Uniform heat distribution was obtained at a body surface with an averaged temperature of $33^{\circ} \mathrm{C}$ as shown in Fig. 4(d). One minute after bathing, ECG readings were severely disturbed with a spike in heart rate to $131.7 \mathrm{bpm}$. Subsequently, the heart rate dropped sharply and ranged from 80 to $90 \mathrm{bpm}$. The SNRs of the R and T waves were 21.5 and $17.5 \mathrm{~dB}$, respectively, almost the same as before bathing. The body surface temperature of around $30^{\circ} \mathrm{C}$ was measured immediately after leaving the bath, which was lower than before bathing, and uneven temperature distribution was observed. Immediately after leaving the bath, the heart rate was found to be high at $101 \mathrm{bpm}$. Over time, it dropped to $70-80 \mathrm{bpm}$, the same as before bathing. The SNRs of the $\mathrm{R}$ and $\mathrm{T}$ waves were 21.7 and $17.5 \mathrm{~dB}$, respectively, and no definite change was observed.

In this experiment, the SNRs of the $\mathrm{R}$ and $\mathrm{T}$ waves were always constant. This result suggests that ECG was measured in warm water as in the atmosphere. The heart rate showed a sharp rise at two points. They would relate to a rapid temperature change both immediately after bathing and immediately after leaving the bath. Rapid changes in heart rate have been associated with the risk of heart failure. ${ }^{[23]}$ This experiment clarified the period during bathing when the risk of the body was high, such as arrhythmia, myocardial infarction, using a device that placed less burden on the patient.

Regarding our study's limitations, daily biosensing with high quality of life requires all components (including circuits, antennas, and batteries) to be composed of soft materials, miniaturized or thinned. Besides, by using a nanosheet made of a material having a high seal property instead of Cathereeplus $^{\mathrm{TM}}$, it is expected that high durability and comfortable can be obtained.

Combining waterproofness and comfort is one of the significant challenges in the current expansion of biosensing applications. We hope that this folded conductive nanosheet will significantly contribute to a broader range of biosensing applications.

\section{Conclusions}

FNE, which is thin and conformable, exhibited high stability in warm water. This electrode enabled ECG measurements during bathing, currently a challenge to measure with existing gel electrodes. ECG measurements during bathing could clarify the points at which the heart rate rose sharply, suggesting the presence of particularly high-risk periods during this activity. FNE can be applied to monitor bathing for high-risk patients with heart disease. Moreover, we believe that FNE will function as part of the biological information monitoring apparatus used in everyday life.

\section{Supplementary material}

The supplementary material for this article can be found at https://doi.org/10.1557/mrc.2020.72.

\section{Acknowledgments}

This work was supported by Waseda Research Institute for Science and Engineering Project Research (Projects number: 20P15). The authors would like to thank Ms. Adeline Koh Mei Choo at Singapore Polytechnic for English language editing, ASAHI RUBBER INC., ASAHI FR Co. Ltd. and Mr. Tatsuto Nakajima for assistance with ECG measurements.

\section{References}

1. S. Kwon, Y. Kwon, Y. Kim, H. Lim, M. Mahmood, and W. Yeo: Skin-conformal, soft material-enabled bioelectronic system with minimized motion artifacts for reliable health and performance monitoring of athletes. Biosens. Bioelectron. 151, 111983 (2020).

2. A.J. Bandodkar, W. Jia, and J. Wang: Tattoo-based wearable electrochemical devices: A review. Electroanalysis 27, 562-572 (2015).

3. S.L. Colyer and P.M. McGuigan: Textile electrodes embedded in clothing: A practical alternative to traditional surface electromyography when 
assessing muscle excitation during functional movements. J. Sports Sci. Med. 17, 101-109 (2018).

4. A.S. Pentland: Healthwear: Medical technology becomes wearable. IEEE Comput. Soc., 37, 42-49 (2004).

5. S. Majumder, T. Mondal, and M.J. Deen: Wearable sensors for remote health monitoring. Sensors 17, 130 (2017).

6. S. Keesara, A. Jonas, and K. Schulman: Covid-19 and health care's digital revolution. N. Engl. J. Med. 382, 1-2 (2020).

7. K. Hung, Y.T. Zhang, and B. Tai: Wearable medical devices for tele-home healthcare. Annu. Int. Conf. IEEE Eng. Med. Biol. Proc. 26, 5384-5387 (2004).

8. B.A. Reyes, H.F. Posada-Quintero, J.R. Bales, A.L. Clement, G.D. Pins, A. Swiston, J. Riistama, J.P. Florian, B. Shykoff, M. Qin, and K.H. Chon: Novel electrodes for underwater ECG monitoring. IEEE Trans. Bio-Med. Eng. 61, 1863-1876 (2014).

9. M. Sessolo, D. Khodagholy, J. Rivnay, F. Maddalena, M. Gleyzes, E. Steidl, B. Buisson, and G.G. Malliaras: Easy-to-fabricate conducting polymer microelectrode arrays. Adv. Mater. 25, 2135-2139 (2013).

10. A. Jonsson, S. Inal, I. Uguz, A. Williamson, L. Kergoat, J. Rivnay, D. Khodagholy, M. Berggren, C. Bernard, G.G. Malliaras, and D.T. Shimon: Bioelectronic neural pixel: Chemical stimulation and electrical sensing at the same site. Proc. Natl. Acad. Sci. USA 113, 9440-9445 (2016).

11. Q. Wang, X. Pan, C. Lin, D. Lin, Y. Ni, L. Chen, L. Huang, S. Cao, and X. Ma: Biocompatible, self-wrinkled, antifreezing and stretchable hydrogelbased wearable sensor with PEDOT:sulfonated lignin as conductive materials. Chem. Eng. J. 370, 1039-1047 (2019).

12.S. Tsukada, H. Nakashima, and K. Torimitsu: Conductive polymer combined silk fiber bundle for bioelectrical signal recording. PLOS One 7 , e33689 (2012).

13. A. Zucca, K. Yamagishi, T. Fujie, S. Takeoka, V. Mattoli, and F. Greco: Roll to roll processing of ultraconformable conducting polymer nanosheets. J. Mater. Chem. C 3, 6539-6548 (2015).

14. K. Yamagishi, S. Takeoka, and T. Fujie: Printed nanofilms mechanically conforming to living bodies. Biomater. Sci. 7, 520-531 (2019).

15. Y. Tetsu, K. Yamagishi, A. Kato, Y. Matsumoto, M. Tsukune, Y. Kobayashi, M.G. Fujie, S. Takeoka, and T. Fujie: Ultrathin epidermal strain sensor based on an elastomer nanosheet with an inkjet-printed conductive polymer. Appl. Phys. Express 10, 1-4 (2017).

16. H. Okuzaki: Hierarchical Structure of PEDOT/PSS and Applications to Transparent Electrodes. Proc. 19th Int. Work. Act. Flatpanel Displays Devices - TFT Technol. FPD Mater. (AM-FPD) 2012; pp. 53-56.

17. T. Chiba, M. Yamauchi, N. Nishida, T. Kaneko, K. Yoshizaki, and N. Yoshioka: Risk factors of sudden death in the Japanese hot bath in the senior population. Forensic Sci. Int. 149, 2-3 (2005).

18. J. Guerreiro, R. Martins, H. Silva, A. Lourenço, and A. Fred: BITalino: A multimodal platform for physiological computing. In ICINCO $2013-$ Proc. 10th Int. Conf. Informatics Control. Autom. Robot, 1, 2013; pp. 500-506.

19. H.U. Li, and T.N. Jackson: Flexibility testing strategies and apparatus for flexible electronics. IEEE Trans. Electron Devices 63, 1934-1939 (2016).

20. N. Sato, A. Murata, T. Fujie, and S. Takeoka: Stretchable, adhesive and ultra-conformable elastomer thin films. Soft Matter 12, 9202-9209 (2016).

21. R. Teraoka, M. Miyake, M. Itoh, T. Shiono, C. Numata, M. Nakayama, Y. Okamoto, M. Hirai, R. Yutani, S. Kitagawa, and T. Sakane: Half dose administration using once-a-day transdermal patch of fentanyl citrate with film dressings. Japanese J. Pharm. Health Care Sci. 43, 671-679 (2017).

22. M. KuŞ, and S. Okur: Electrical characterization of PEDOT:PSS beyond humidity saturation. Sensors Actuat. B Chem. 143, 177-181 (2009).

23. M.W. Gillman, W.B. Kannel, A. Belanger, and R.B. D'Agostino: Influence of heart rate on mortality among persons with hypertension: The framingham study. Am. Heart J. 125, 1148-1154 (1993). 